\title{
Repeated pupillary capture and pigmentary glaucoma after implantation of reversed three-piece sulcus intraocular lens
}

\author{
Ha-Uyen T. Nguyen, ${ }^{1}$ Elizabeth Yeu-Lin, ${ }^{1,2}$ Albert P. Lin ${ }^{1,2}$ \\ ${ }^{1}$ Baylor College of Medicine; ${ }^{2}$ Michael E DeBakey Veterans Affairs Medical Center, \\ Houston, Texas, USA
}

\begin{abstract}
A 58-year-old man underwent phacoemulsification, anterior vitrectomy, and a reversed three-piece intraocular lens placement in the sulcus. He developed pseudophakic pupillary block with optic capture of the intraocular lens four months later and underwent intraocular lens repositioning combined with surgical iridectomy. After 18 months, he had elevated intraocular pressure that was thought to be secondary to complicated cataract surgery, and he was prescribed topical glaucoma medications. He had poor adherence and follow-up attendance. After another six months, he presented to the Baylor College of Medicine, Houston, USA, with an intraocular pressure of $30 \mathrm{mmHg}$ and advanced pigmentary glaucoma. The intraocular lens optic was captured by the pupil after dilation. A trabeculectomy and intraocular lens exchange was performed. Reversed intraocular lens in the sulcus may present as repeated intraocular lens pupillary capture and result in the development of pigmentary glaucoma.
\end{abstract}

Key words: Phacoemulsification, lenses, intraocular, intraocular pressure, glaucoma, trabeculectomy

\section{Introduction}

Pseudophakic pupillary block is a known complication of reversed intraocular lens $(\mathrm{IOL})^{1,2}$ and there have been reports of IOL repositioning with good results. ${ }^{2}$ However, there have been no reports of repeat presentation after repositioning. This report is of a patient with a reversed sulcus IOL presenting with repeated pupillary capture of the IOL optic associated with the development of advanced pigmentary glaucoma.

\section{Case report}

A 58-year-old man presented in 2008 with visually significant cataract in the left eye and best-corrected visual acuity (BCVA) of 20/70, intraocular pressure (IOP) of $16 \mathrm{mmHg}$, and cup-disc ratio of 0.55 . He underwent phacoemulsification cataract extraction and had posterior capsular rupture at another institution. Anterior vitrectomy was performed, followed by sulcus placement of a 17.0 D MA60AC IOL (Alcon Laboratories, Inc, Fort Worth, Texas, USA) 3-piece IOL with prolen haptics ( $13 \mathrm{~mm}$ length, $6 \mathrm{~mm}$ optics, $10^{\circ}$ posterior vault). One month after surgery, his BCVA was 20/30 and IOP was $17 \mathrm{mmHg}$.

Four months postoperatively, the patient developed sudden-onset pain and blurred vision. Ocular examination revealed vision of counting fingers, microcystic corneal oedema, IOP of $34 \mathrm{mmHg}$, and pseudophakic pupillary block with pupillary capture of

Correspondence: Dr Albert P. Lin, 2002 Holcombe Boulevard 112C, Houston, Texas 77030, USA. E-mail: alin@bcm.edu. 
the IOL. The patient was given topical and oral aqueous suppressants and the IOL was repositioned and surgical iridectomy was performed. Two months later, his BCVA was 20/30 (-1.75 + $0.50 \times 118)$, IOP was $14 \mathrm{mmHg}$, and cup-disc ratio was 0.6. Glaucoma suspect status was diagnosed given the enlarged cup-disc ratio. A baseline Humphrey visual field was unreliable.

After 18 months, the patient was found to have an IOP of $23 \mathrm{mmHg}$ and an increased cup-disc ratio of 0.75 . He was diagnosed with secondary glaucoma with trabecular dysfunction from complicated cataract surgery and was given travoprost and combination timolol and dorzolamide. His IOP improved to $17 \mathrm{mmHg}$.

Six months after this visit, the patient presented to the Baylor College of Medicine, Houston, USA, for evaluation. Examination showed stable vision, relative afferent pupillary defect, IOP of $30 \mathrm{mmHg}$, diffuse pigments on the corneal endothelium, 4+ pigments in the chamber angle (Fig. 1), and cup-disc ratio of 0.85 . Humphrey visual field demonstrated dense arcuate defects involving the fixation. He was not using his drops and medical therapy was restarted. At the follow-up visit, his IOP was $22 \mathrm{mmHg}$ and he was

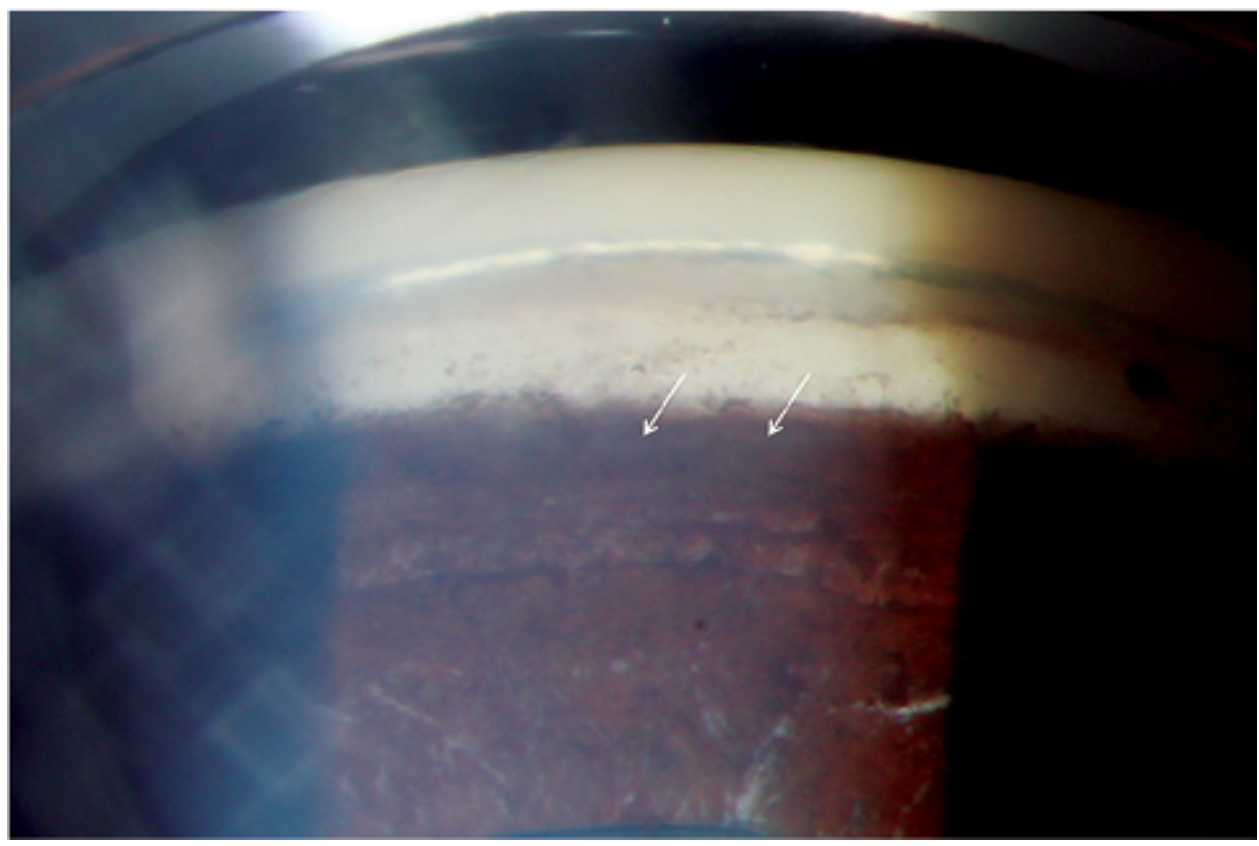

Fig. 1. Examination of the inferior chamber angle shows dense pigment deposition. The scleral spur is barely visible (arrows).

found to have pupillary capture of the IOL optic. His BCVA was 20/25 (-2.00 sphere) and the remaining findings were unchanged. The haptic was pointing in a clockwise direction (S-shaped; Fig. 2) and a diagnosis of a reversed sulcus IOL was made. Combined trabeculectomy with mitomycin $\mathrm{C}$ and IOL exchange was performed. The reversed intraocular lens was removed, additional anterior vitrectomy was performed, and the 
IOL was replaced with a sulcus 17.5 D Alcon MA60AC. One month after surgery, his BCVA was $20 / 25+1(-0.75+0.50 \times 105)$ and the IOP was six $\mathrm{mmHg}$. The IOL was found to be in a good position with and without dilation (Fig. 3).

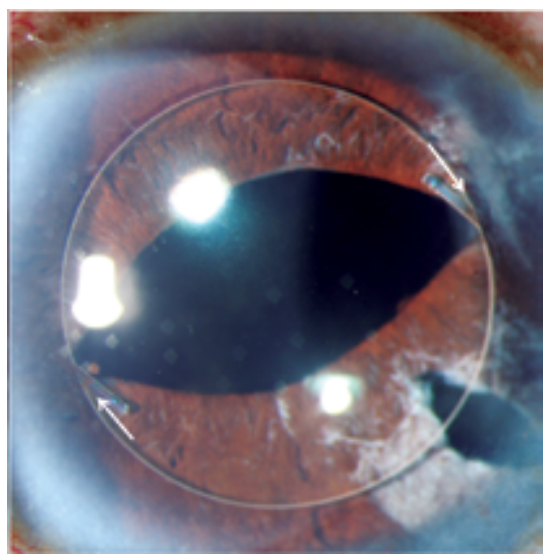

Fig. 2. Pupillary capture of the intraocular lens optic with haptics in the sulcus. The haptics were pointing clockwise (arrows), indicating that the intraocular lens was reversed.

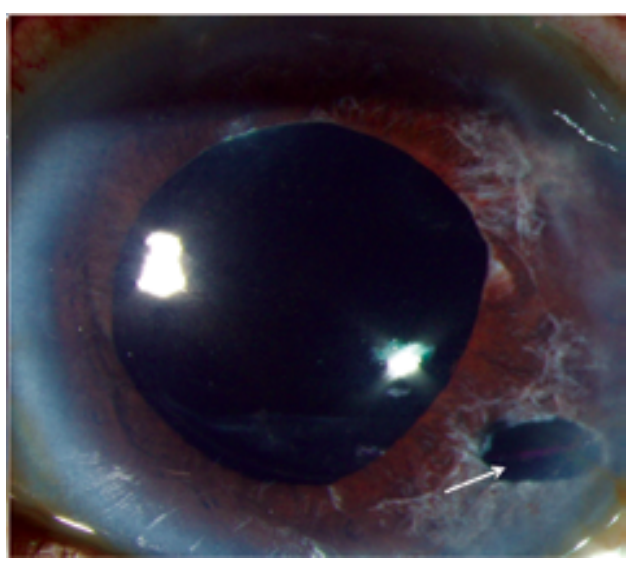

Fig. 3. The intraocular lens was stable on dilation after exchange and trabeculectomy. The haptics were pointing counter clockwise (arrow), indicating that the intraocular lens was in the correct orientation.

\section{Discussion}

Pseudophakic pupillary block with IOL placement in the capsular bag or sulcus has been described and managed with IOL repositioning, either non-invasively or surgically.1-5 Secondary glaucomas, such as pigmentary glaucoma and uveitis glaucoma hyphaema (UGH) syndrome, are well-known problems associated with sulcus IOL placement. ${ }^{6,7}$ This report describes a patient with unrecognized reversed sulcus IOL placement that presented with repeated pupillary capture of the IOL optic and advanced pigmentary glaucoma despite previous repositioning and iridectomy. The authors did not observe the IOL capture until the second visit, presumably because the IOL only moved forward subsequent to the dilation at the first visit.

Potential problems with reversed sulcus IOL include pseudophakic pupillary block, secondary glaucoma, and changes in effective IOL power. Pseudophakic pupillary block may have resulted in this patient because the posterior vault of the lens resulted in anterior displacement of the lens optic towards the pupil when it was reversed. When the IOL is placed in the capsular bag, the angulation may decrease over time due to scarring of the anterior and posterior capsule, but this process does not occur when the lens is placed in the sulcus. If residual vitreous was still present behind the $\mathrm{OOL}$, additional posterior pressure may also have contributed to this movement. Pupillary block did not occur when the IOL optic was captured by the pupil the second time because of the patent iridectomy.

Secondary glaucoma can occur from optic or haptic contact with adjacent ocular 
structures such as the iris, ciliary body, or vasculature. This patient most likely had pigment liberation from the iris, as evidenced by the amount of pigment deposited on the corneal endothelium and chamber angle. Excessive pigment deposition may lead to trabecular dysfunction and elevated IOP over time.

Changes in effective lens power may come from changes in IOL position as well as the anterior and posterior radii of curvature of the IOL. The IOL position moved anteriorly away from the retina in this patient because of the sulcus placement, anterior vault, and optic capture by the pupil, which all contributed to an increase in effective lens power and a shift towards myopia. The intended aim of the original cataract surgery was not known, but the patient's refraction changed from -1.50 to -2.00 spherical equivalent when he presented with the IOL optic in the anterior chamber. Postoperatively, even though the IOL power was increased by +0.50 sphere, refractive error decreased to -0.50 spherical equivalent because pupillary capture was no longer present and the IOL now had a posterior vault. The Alcon MA60AC is an anterior biconvex $\mathrm{IOL}$ with similar anterior and posterior radii of curvature. In an average eye, a reversed $\mathrm{IOL}$ of this type will result in a negligible change in postoperative refractive error $(-0.05$ to -0.34 sphere in an eye with $23 \mathrm{~mm}$ axial length). ${ }^{8}$ It is interesting to note the patient did not have significant inflammation, synechiae formation, or iris transillumination defects despite the large amount of pigment observed on the cornea and chamber angle. After the surgery, the patient had excellent vision and IOP without choroidal effusion.

A reversed sulcus IOL may result in pseudophakic pupillary block, secondary glaucoma, and unintended postoperative myopia. When a patient presents with a sulcus IOL and repeated pupillary optic capture, a reversed IOL should be suspected, although findings of a reversed IOL may be subtle and may sometimes be missed at initial presentation. IOL exchange combined with trabeculectomy was indicated in this patient because of the repeat presentation despite IOL repositioning, patent iridectomy, and the need to control IOP in the presence of advanced pigmentary glaucoma.

\section{References}

1. Awan MA, Choi J. Reversed sulcus-fixated posterior chamber intraocular lens leading to pupillary capture. Asian J Ophthalmol 2006;8:151-152.

2. Harsum S, Low S. Reversed vaulted AcrySof intraocular lens presenting as pupillary block. Eye 2009;23:1880-1882.

3. Galvis V, Tello A, Montezuma S. Delayed pupillary capture and noninvasive repositioning of a posterior chamber intraocular lens after pupil dilation. J Cataract Refract Surg 2002;28:1876-1879.

4. Nagamoto S, Kohzuka T, Nagamoto T. Pupillary block after pupillary capture of an AcrySof intraocular lens. J Cataract Refract Surg 1998;24:1271-4.

5. Khokhar S, Sethi HS, Sony P, Sudan R, Soni A. Pseudophakic pupillary block caused by pupillary capture after phacoemulsification and in-the-bag AcrySof lens implantation. J Cataract Refract Surg 2002;28:1291-1292.

6. Allingham RR, Damji KF, Freedman S, Moroi SE, Shafranov G. Shield's Textbook of glaucoma. 5th ed. Philadelphia: Lippincott Williams \& Wilkins 2005; pp. 416-417.

7. Almond MC, Wu MC, Chen PP. Pigment dispersion and chronic intraocular pressure elevation after sulcus placement of 3-piece acrylic intraocular lens. J Cataract Refract Surg 2009;35:2164-2166.

8. Nawa, Y, Okamoto M, Tsuji H, Ueda T, Kanzaki M, Hara Y. Dioptric changes in eyes with reversed intraocular lenses. J Cataract Refract Surg 2005;31:586-589. 